\title{
Tissue laser biostimulation promotes post-extraction neoangiogenesis in HIV-infected patients
}

\author{
Agnieszka Halon • Piotr Donizy • Mateusz Dziegala • \\ Rafal Dobrakowski $\cdot$ Krzysztof Simon
}

Received: 23 April 2013 / Accepted: 22 July 2013 /Published online: 6 August 2013

(C) The Author(s) 2013. This article is published with open access at Springerlink.com

\begin{abstract}
The aim of the study was to assess the rate of neoangiogenesis in extraction wound healing following exposure to biostimulating laser therapy and to analyze the correlation between parameters of neoangiogenesis as reflected by the number and surface area of newly formed blood vessels and clinical parameters such as gender, position of a tooth in the oral cavity, and CD4 lymphocyte count. Twenty-seven patients with confirmed HIV infection were enrolled in the study (6 women, 21 men). Eighty-nine teeth were extracted; 45 sockets were exposed to $6 \mathrm{~J}$ laser radiation (laser parameters were set as follows: wavelength, $820 \mathrm{~nm}$; output, $200 \mathrm{~mW}$; dose, $6 \mathrm{~J} / \mathrm{cm}^{2}$; spot size, $38 \mathrm{~mm}^{2}$; continuous radiation) for five consecutive days following tooth extraction, and the remaining extraction wounds were left to heal spontaneously without laser irradiation. Antigen CD34 was assessed by immunohistochemistry as a marker of angiogenesis, and its expression was examined by computer-assisted histomorphometric image analysis. As a result, we report that biostimulating laser therapy in HIV-infected patients of varying degrees of immunodeficiency greatly accelerated postextraction neoangiogenesis, regardless of the patient's gender, tooth position, number of roots, or number of CD4 lymphocytes in the blood. Application of low-level laser therapy for the treatment of tooth extraction wounds in $\mathrm{HIV}(+)$ patients greatly enhanced the formation of new blood vessels, which in turn promoted wound healing.
\end{abstract}

\footnotetext{
A. Halon $(\varangle) \cdot$ P. Donizy $\cdot$ M. Dziegala

Department of Pathomorphology and Oncological Cytology,

Wroclaw Medical University, Borowska 213,

50-556 Wroclaw, Poland

e-mail: ahalon2@gmail.com

R. Dobrakowski $\cdot$ K. Simon

Division of Infectious Diseases and Hepatology, Wroclaw Medical

University, Koszarowa 5, 51-149 Wroclaw, Poland
}

Keywords Human immunodeficiency · Low-level laser therapies $\cdot$ Biostimulation $\cdot$ Tooth extraction

\section{Introduction}

An estimated 33 million people worldwide live with HIV, with almost $97 \%$ of them living in developing countries [1]. HIV infection remains asymptomatic for a long period of time, and clinical manifestation is subtle and nonspecific. However, the virus systematically destroys the immune system, including the CD4+ T lymphocytes of the infected individual [2].

Oral lesions are one of the first symptoms reported by people living with HIV/AIDS, and apart from the symptoms of acute HIV infection, they are often the first clinical manifestation of the disease $[3,4]$.

The likelihood of rendering care to HIV-infected people at a dental clinic is high even though very few people declare their HIV-positive status. The oral cavity should be routinely examined for HIV-related oral lesions and signs of the fullblown AIDS which most often involve oral candidiasis, hairy leukoplakia, and several types of periodontal diseases [5-7]. HIV-infected patients also face more serious health issues that require professional dental intervention and care.

Laser biostimulation is a proven and safe form of therapy [8]. One of the main advantages of biostimulating laser is increase in the activity and number of $\mathrm{T}$ lymphocytes that produce cytokines uniquely responsible for tissue regeneration. Increase in lymphocyte activity stimulates phagocytic activity of macrophages [9], which is of primary importance for wound healing in the oral cavity inhabited by a large number of potential pathogens $[10,11]$. Laser radiation also promotes fibroblast activity and stimulates collagen synthesis, which accelerates connective tissue wound healing and ensures better properties of newly formed collagen. Acceleration of tissue regeneration induced by laser biostimulation is associated with neoangiogenesis activation and the resulting increase in tissue oxygen partial pressure, which in turn enhances mitosis [12]. 
The aim of the present study was to assess neoangiogenesis process following extraction of a tooth in HIV-positive patients. Sockets were selected at random and subjected to low-level laser radiation so that some of them were healed after exposure to laser radiation and others healed spontaneously. Tissue specimens were collected from the patient's oral mucosa 5 days following dental surgery. Correlations between post-extraction neoangiogenesis reflected by surface area and the number of newly formed blood vessels and clinical parameters such as gender, position of a tooth in the mouth, sector in the oral cavity, and the number of CD4 lymphocytes were analyzed.

\section{Materials and methods}

\section{Patients}

Twenty-seven patients were enrolled in the study, including 6 women and 21 men with confirmed HIV infection. Due to varied dental reasons (advanced decay, strong tooth loosening caused by inflammation of periodontal tissues), 89 teeth were extracted -22 teeth from the maxilla and 67 from the mandible. Informed consent was obtained from all participants. The experiment was approved by the Institutional Review Board at the Wroclaw Medical University, Wroclaw, Poland. HIVinfected patients were grouped according to the 1993 CDC Classification System [13]: seven patients with CD4 lymphocyte count $>500 / \mathrm{ml}$, eight patients with CD4 lymphocyte count in the range of 200 to $499 / \mathrm{ml}$, and seven patients with CD4 lymphocyte count $<200 / \mathrm{ml}$. Due to clot formation in blood specimens, in five patients the number of CD4 lymphocytes was impossible to determine.

\section{Procedure}

The device used in the study was Doris CTL 1106MX (diode laser; output, 5-250 mW; wavelength, $820 \mathrm{~nm}$ ) by Laser Instruments (Warsaw, Poland). Laser parameters were set as follows: wavelength, $820 \mathrm{~nm}$; output, $200 \mathrm{~mW}$; dose, $6 \mathrm{~J} / \mathrm{cm}^{2}$; and spot size, $38 \mathrm{~mm}^{2}$. Forty-five sockets were exposed to laser radiation, and the remaining 44 post-extraction wounds healed spontaneously with no additional perioperative or postoperative treatment. All except two of the patients' sockets were subjected to both low-level laser therapy and left to heal spontaneously. The adjacent sites were not treated with laser. No additional topical treatment was applied in the perioperative or postoperative period. None of the wounds was surgically closed. No pharmacological protocol was introduced after the extractions.

The procedure adopted in the study involved the following: (1) tooth extraction preceded by clinical examination and identification of reasons for extraction, (2) continuous radiation with a biostimulating laser prober for five consecutive days following tooth extraction at a dose of $6 \mathrm{~J} / \mathrm{cm}^{2} /$ day and
$200 \mathrm{~mW} / \mathrm{day}$, and (3) sampling the oral mucosa (by fineneedle aspiration biopsy) of the healing socket for immunohistochemical examination on the sixth day following extraction.

Immunohistochemistry

Specimens sampled from the studied patients were fixed in $10 \%$ buffered formalin and embedded in paraffin. In every case, hematoxylin and eosin stained sections were examined by two pathologists.

Formalin-fixed, paraffin-embedded tissue was freshly cut $(4 \mu \mathrm{m})$. For the detection of CD34, a monoclonal mouse antibody (Clone PC10, Novocastra, UK) was diluted 1:100 in Antibody Diluent with Background Reducing Components (DakoCytomation, Poland). Tested sections were incubated with antibodies for $1 \mathrm{~h}$ at room temperature. Subsequent incubations involved biotinylated antibodies (15 min, room temperature) and streptavidin-biotinylated peroxidase complex (15 min, room temperature) (LSAB+, HRP, DakoCytomation, Poland). NovaRed (Vector Laboratories, UK) was used as a chromogen (10 $\mathrm{min}$, at room temperature). All the sections were counterstained with Meyer's hematoxylin. In each case, control reactions were included, in which a specific antibody was substituted by the Primary Mouse Negative Control (DakoCytomation, Poland).

\section{Angiogenesis intensity}

Angiogenesis was assessed by CD34 antigen immunohistochemical expression using an optical microscope. The microscopic image was analyzed using MultiScaneBase. A semiquantitative analysis was used to assess CD34 antigen expression. The microscopic image obtained from a specimen was magnified $400 \times$ and transferred to a computer program, and all vessels stained positive were counted automatically for five randomly selected fields of view with the highest microvessel density. Every single endothelial cell or a cluster of cells was considered a "hot point" and counted as an individual microvessel. For each specimen (five fields), the number of vessels $(\mathrm{N})$ and vessel surface area $(\mathrm{SA})$ were calculated.

\section{Statistical analysis}

To estimate the differences in the mean values of the examined features in the two groups, Student's $t$ test was used for independent samples of unequal or similar variations. The variance differences for two samples were determined by $F$ test. $P$ value below which variables are statistically significant was 0.05 . To estimate the differences in the mean values of the examined features for more than two independent groups, one-way analysis of variance was used. To examine the influence of two factors on the examined features, two-way analysis of variance was used. 


\section{Results}

Correlations between immunohistochemical parameters of angiogenesis and patients' sex and dental status

The experimental group was predominantly men (84\%). The average number of sockets subjected to treatment was almost three times higher in men than in women. Most sockets were examined in sector 4 , followed by sector 3 .

No statistically significant difference was found in comparing the vessel surface area (SA1-SA5) and the number of blood vessels (N1-N5) in the group of HIV-infected women and men that underwent dental surgery $(P>0.05)$. Moreover, no significant correlations were observed between vessel surface area and the number of blood vessels, and the tooth sector $(P>0.05)$ (Table 1).

Correlations between angiogenesis intensity and applied laser therapy

In each of the five randomly selected fields, vessel surface area was significantly higher in patients exposed to radiation. The differences were statistically significant for all fields $(P=0.0002)$ (Table 2).

Moreover, we demonstrated that the number of vessels is much higher in areas exposed to radiation than in those not subjected to low-level laser therapy. In all areas, the observed increase in the number of vessels was statistically significant $(P=0.0003)$ (Table 2, Fig. 1).

Analysis of correlations between laser therapy, angiogenesis intensity, and selected clinical and dental features

Statistical analysis of the correlation between the mean values and standard deviations of vessel surface area and the number of vessels in the three groups formed based on the number of CD4 lymphocytes in the blood (over 500/ml, 499-200/ml, and below 200/ml) showed no statistically significant differences $(P>0.5)$. It is likely that the effect of radiation is not dependent on the number of CD4 lymphocytes, which have been shown to not accelerate neoangiogenesis, as reflected by the vessel surface area and the number of vessels (Fig. 2a, b).

Moreover, analysis of variance examining the influence of radiation and position of the tooth in the maxilla or mandible on the socket healing process showed that only exposure to radiation significantly enhances the neoangiogenesis process (Fig. 2c, d).

Another aspect that was analyzed was the correlation between the rate of post-extraction neoangiogenesis and exposure to radiation and the number of tooth roots (multi-rooted/singlerooted teeth). Figure 2e, f gives the result of two-way analysis of variance examining the significance of exposure to radiation and the number of roots on the surface area and the number of blood vessels. It was demonstrated that exposure to radiation was the only factor of significance, while the number of roots had no effect on the rate of neoangiogenesis parameters.

\section{Discussion}

In contrast to surgically closed wounds that follow a predictable sequence of events, post-extraction wounds involve considerable tissue loss that is replaced by granulation tissue in a non-sterile environment. HIV-positive patients involved in the study, due to varying degrees of immunodeficiency, faced a higher risk of post-extraction complications.

Although several studies have been reported, the correlation between HIV infection and wound healing process remains unclear. Harrison et al. [14] performed a prospective, controlled study on wound infection after implant surgery involving 41 HIV-positive and 141 HIV-negative patients. The CD4 cell count had no influence on the incidence of infections. The main conclusion was that that when no contamination has occurred, the wound healing is within the normal limits and implant

Table 1 Patient characteristics and correlation between tooth sector in the oral cavity and parameters of angiogenesis

\begin{tabular}{|c|c|c|c|c|c|c|c|c|c|c|c|c|}
\hline \multirow[t]{2}{*}{ Characteristics of patients } & \multicolumn{6}{|c|}{ Vessel surface area (SA1-SA5), $\mu \mathrm{m}^{2}$} & \multicolumn{6}{|c|}{ Number of blood vessels (N1-N5) } \\
\hline & SA1 & SA2 & SA3 & SA4 & SA5 & $P$ value ${ }^{\mathrm{a}}$ & N1 & $\mathrm{N} 2$ & $\mathrm{~N} 3$ & N4 & N5 & $P$ value $^{\mathrm{a}}$ \\
\hline Men & 7.83 & 8.15 & 6.71 & 6.66 & 7.38 & \multirow[t]{2}{*}{ NS } & 5.35 & 6.03 & 5.35 & 5.49 & 5.45 & \multirow[t]{2}{*}{ NS } \\
\hline Women & 6.45 & 7.29 & 6.64 & 5.73 & 5.03 & & 5.50 & 6.71 & 6.36 & 5.36 & 5.57 & \\
\hline \multicolumn{6}{|l|}{ Sector (number) } & \multirow[t]{5}{*}{ NS } & & & & & & \multirow[t]{5}{*}{ NS } \\
\hline $1(8)$ & 10.19 & 10.34 & 7.76 & 7.45 & 9.00 & & 7.13 & 7.25 & 6.88 & 5.75 & 6.00 & \\
\hline $2(14)$ & 5.79 & 8.62 & 5.68 & 6.67 & 7.71 & & 4.36 & 5.57 & 5.50 & 5.36 & 5.00 & \\
\hline $3(32)$ & 6.30 & 7.24 & 6.96 & 5.74 & 5.71 & & 4.78 & 5.69 & 4.78 & 5.09 & 5.34 & \\
\hline $4(34)$ & 8.98 & 7.95 & 6.62 & 6.96 & 7.48 & & 5.94 & 6.53 & 5.88 & 5.79 & 5.65 & \\
\hline
\end{tabular}

NS nonsignificant

${ }^{\text {a }}$ Value of Student's $t$ test 
Table 2 Correlation between immunoangiogenic parameters and biostimulating laser therapy

\begin{tabular}{llll}
\hline Immunoangiogenic parameters & \multicolumn{2}{l}{ Low-level laser therapy } & \multirow{2}{*}{$P$ value $^{\mathrm{a}}$} \\
\cline { 2 - 3 } & Yes & No & \\
\hline Total number of sockets & 45 & 44 & \\
Vessel surface area (SA) $^{\mathrm{b}}$ & 9.868 & 4.708 & 0.0002 \\
Number of blood vessels (N) $^{\mathrm{b}}$ & 6.887 & 4.416 & 0.0003 \\
\hline
\end{tabular}

${ }^{\text {a }} P$ value of Shapiro-Wilk test

${ }^{\mathrm{b}}$ Average values

surgery may be undertaken safely. Additionally, Aird et al. [15] had demonstrated that HIV infection is not a significant risk factor for acute wound/implant infection.

On the other hand, Davis et al. [16] had indicated that after laparotomy, there was a significantly greater incidence of wound complications and wound breakdown in the HIVpositive than in HIV-negative group. Moreover, Morandi et al. [17] revealed in a prospective study of healing time after hemorrhoidectomy that there is a high incidence of delayed wound healing in HIV-infected patients.

We have demonstrated that the effect of radiation is not dependent on the number of CD4 lymphocytes, which have been shown to not accelerate neoangiogenesis. The result is somewhat surprising and indicates that the weakened immune system as a result of a decreased number of lymphocytes in the blood does not cause delayed healing of extraction socket. However, due to a small number of participants in this study, this finding needs to be further investigated in a larger group.

Studies carried out by Chen et al. [18] that involved 300 patients assessed as regards the number of post-extraction complications showed no statistically significant differences in the frequency of occurrence of complications such as swelling, lockjaw, swallowing problems, inflammatory infiltrations, or need for hospitalization between $\mathrm{HIV}(+)$ and $\mathrm{HIV}(-)$ patients, and they were observed in $37.5 \% \mathrm{HIV}(+)$ patients. Compared with the number of complications in HIV $(-)$ patients (33\%), they were statistically insignificant
$(P=0.79)$. A study carried out by Dodson [19] involving $\mathrm{HIV}(+)$ and $\operatorname{HIV}(-)$ patients $(151$ in total) produced similar results, with a higher but statistically insignificant $(P=0.15)$ number of complications reported: $22.3 \% \mathrm{HIV}(+)$ patients versus $13.3 \% \mathrm{HIV}(-)$ patients. In the author's own material, no serious post-extraction complications were observed in HIV-infected patients undergoing tooth extraction. Irrespective of immunologic and clinical status, the postoperative period could be considered uncomplicated.

Formation of a network of new blood vessels plays a pivotal role in carcinogenesis. Though much is known about cancer vascularity, the researchers' interest rarely extends to pathologies other than cancer (wound healing, inflammations, degenerative changes). Vascular diseases supported by abnormal neoangiogenesis have increased incidence in HIVinfected patients. Caccuri et al. [20] examined the role of the HIV matrix protein p17 in the angiogenesis process. According to their studies, the viral $\mathrm{p} 17$ protein binds with high affinity to CXCR2, which is a CXCR1-related receptor, and promotes the formation of capillary-like structures on human endothelial cells (EC) by interacting with CXCR1 and CXCR2 expressed on the EC surface. Another study, carried out by Albini et al. [21], suggests that under specific circumstances, HIV Tat protein promotes the growth of spindle cells and demonstrates angiogenic properties.

MVD, which is the density of newly formed blood vessels, also proved an important factor in diagnosing various forms of myelodysplastic syndromes (MDSs). The comparison of two groups of patients, $\mathrm{HIV}(+)$ and $\mathrm{HIV}(-)$ individuals, suffering from different forms of MDSs revealed higher intensity of angiogenesis reflected by higher MVD in both groups as compared with the bone marrow of healthy individuals. No correlation was found, like in the group of patients assessed by the author of this paper, between angiogenesis intensity and the number of CD4 lymphocytes or the clinical status of $\mathrm{HIV}(+)$ patients [22].

Our study revealed no statistically significant correlation between the parameters of neoangiogenesis and patients' immunologic status reflected by the number of CD4 lymphocytes, gender, tooth location, or the number of roots. While
Fig. 1 CD34 immunoreactivity in tissue specimens from oral cavity mucosa before (a) and after (b) low-level laser therapy
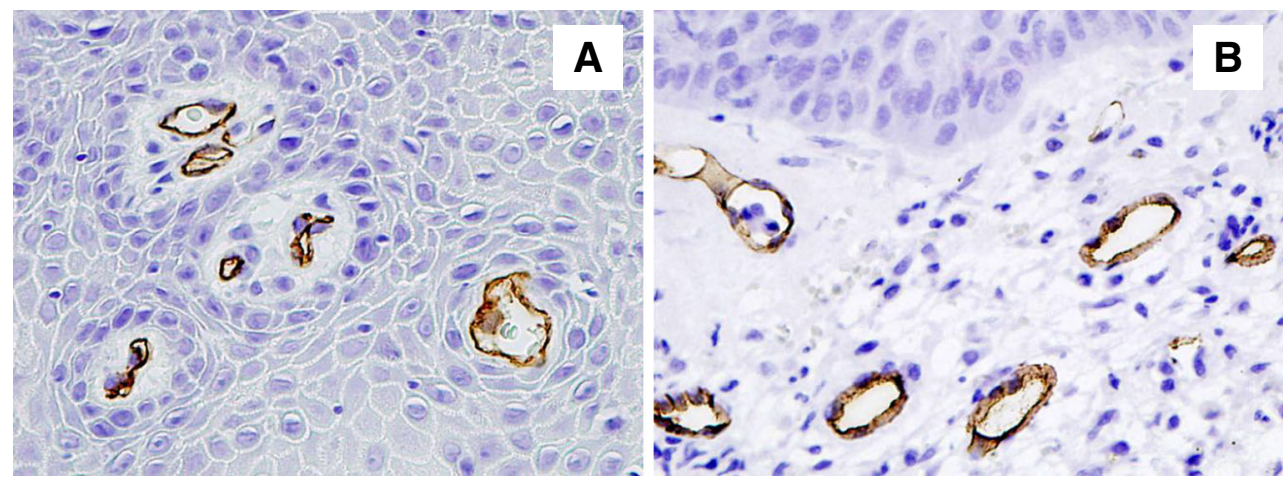
Fig. 2 Comparison of influence of laser therapy and selected clinical and dental features on angiogenesis parameters. $\mathbf{a}, \mathbf{b}$ The mean values of vessel surface area and number of vessels in three groups formed based on the number of CD4 lymphocytes in the blood (over 500/ml, 499-200/ $\mathrm{ml}$, and below $200 / \mathrm{ml}$ ) showed no statistically significant differences $(P>0.05)$ : the effect of radiation is not dependent on the number of CD4 lymphocytes, which have been shown to not accelerate neoangiogenesis, as reflected by the vessel surface area and number of vessels. c, $\mathbf{d}$ Position of the tooth in the maxilla or mandible on the socket healing process showed that only exposure to radiation significantly enhances the neoangiogenesis process. e, f Exposure to radiation was the only factor of significance, while the number of roots had no effect on the rate of neoangiogenesis parameters $(P>0.05)$
A
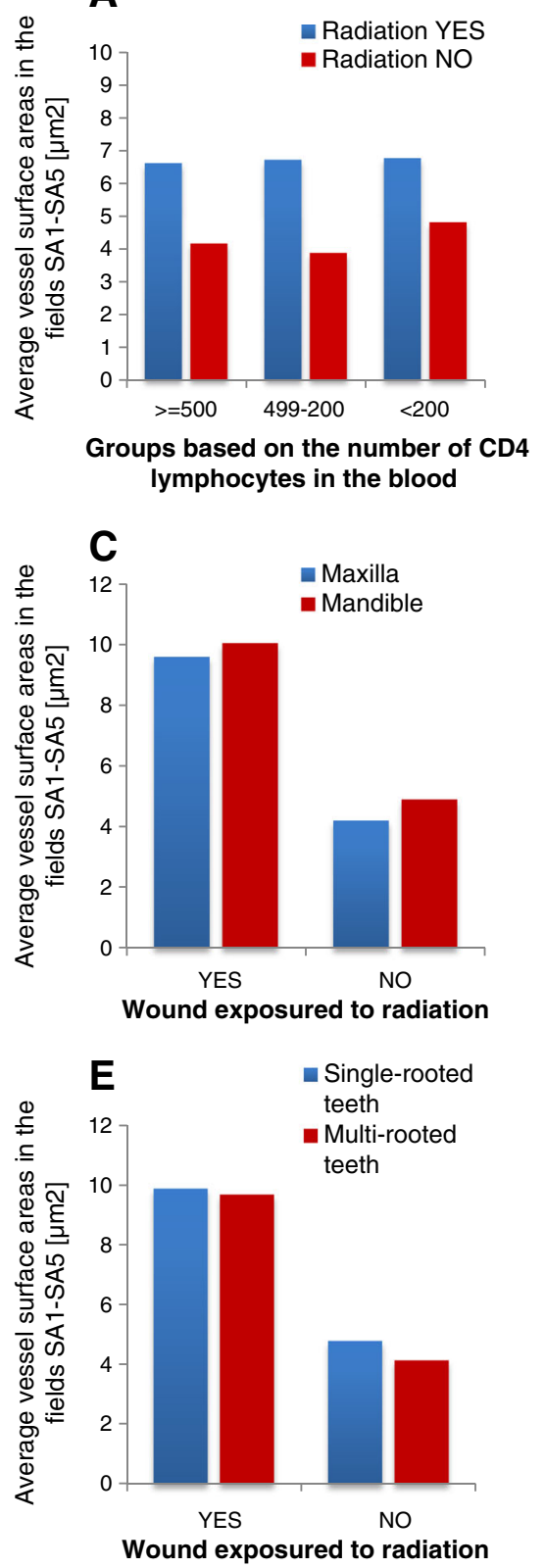

B

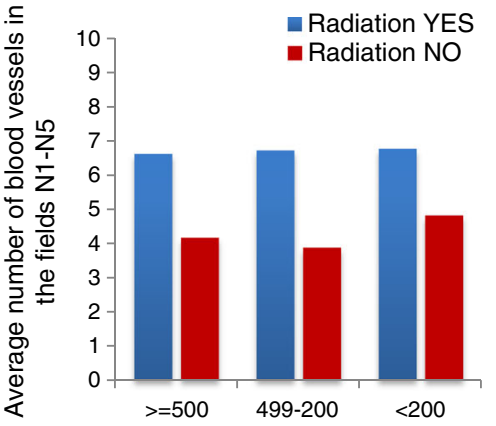

Groups based on the number of CD4 lymphocytes in the blood

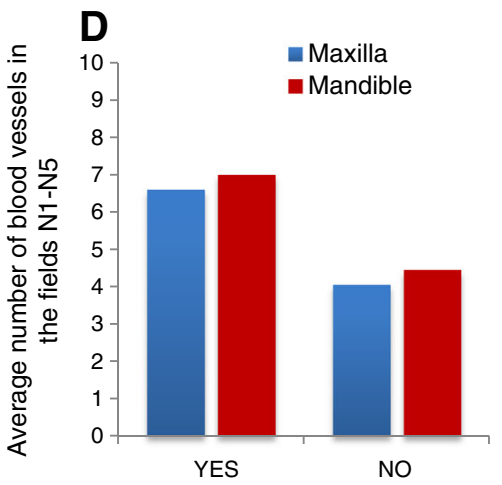

Wound exposured to radiation

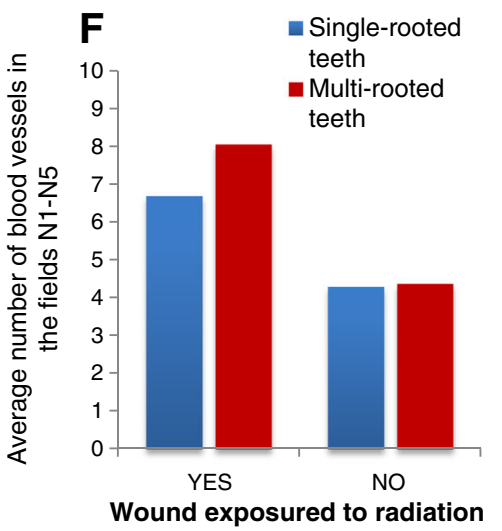

correlations between the number of CD4 lymphocytes in $\mathrm{HIV}(+)$ patients and factors that are widely examined (coinfections and post-extraction complications) are known and frequently discussed, the correlations between MVD and vessel surface area and tooth location, the number of roots, and influence of laser therapy on wound healing process discussed in the present study have not been evaluated in the literature so far.

It is worth examining what MVD and the surface area of newly formed vessels in HIV-infected patients depend on in the recovery process. It seems to be associated with better blood circulation in the tissue during temporary inflammation, which is an initial stage of wound healing cascade. The above is observed not only in persons with acquired immunodeficiency in the course of HIV infection.

In conclusion, the results of our study shed new light on the form of dental care provided to HIV-infected patients. It was demonstrated that the application of low-level laser therapy for the treatment of tooth extraction wounds in $\mathrm{HIV}(+)$ patients greatly enhances the formation of new blood vessels, which in turn promotes wound healing. Accelerated postextraction neoangiogenesis in $\mathrm{HIV}(+)$ patients is an important element of dental treatment as it significantly reduces the incidence of serious extraction healing complications in a high-risk group of patients with acquired immunodeficiency syndrome. 
Conflict of interests We declare no conflict of interest.

Open Access This article is distributed under the terms of the Creative Commons Attribution License which permits any use, distribution, and reproduction in any medium, provided the original author(s) and the source are credited.

\section{References}

1. UNAIDS, the Joint United Nations Programme on HIV/AIDS (2011). Global HIV/AIDS response. Epidemic update and health sector progress towards Universal Access - Progress Report 2011. www.unaids.org

2. Nesti M, Carli E, Giaquinto C, Rampon O, Nastasio S, Giuca MR (2012) Correlation between viral load, plasma levels of CD4-CD8 T lymphocytes and AIDS-related oral diseases: a multicentre study on $30 \mathrm{HIV}+$ children in the HAART era. J Biol Regul Homeost Agents 26:527-537

3. Umeizudike KA, Savage KO, Ayanbadejo PO, Akanmu SA (2011) Severe presentation of necrotizing ulcerative periodontitis in a Nigerian HIV-positive patient: a case report. Med Princ Pract 20:374-376

4. Feller L, Khammissa RA, Wood NH, Meyerov R, Pantanowitz L, Lemmer J (2012) Oral ulcers and necrotizing gingivitis in relation to HIV-associated neutropenia: a review and an illustrative case. AIDS Res Hum Retrovir 28:346-351

5. Reichart PA (2003) Oral manifestations in HIV infection: fungal and bacterial infections, Kaposi's sarcoma. Med Microbiol Immunol 192:165-169

6. Patel AS, Glick M (2003) Oral manifestations associated with HIV infection: evaluation, assessment, and significance. Gen Dent 51:153156

7. Goto M, Onizawa K, Yanagawa T, Yamagata K, Shinozuka K, Nishikii $\mathrm{H}$ et al (2012) Human immunodeficiency virus-associated Burkitt's lymphoma in oral cavity of Japanese patient. J Oral Maxillofac Surg 70:1885-1890

8. Kim SJ, Kang YG, Park JH, Kim EC, Park YG (2012) Effects of lowintensity laser therapy on periodontal tissue remodeling during relapse and retention of orthodontically moved teeth. Lasers Med Sci $28: 325-333$
9. Posten W, Wrone DA, Dover JS, Arndt KA, Silapunt S, Alam M (2005) Low-level laser therapy for wound healing: mechanism and efficacy. Dermatol Surg 31:334-340

10. Amorim JC, de Sousa GR, de Barros SL, Prates RA, Pinotti M, Ribeiro MS (2006) Clinical study of the gingiva healing after gingivectomy and low-level laser therapy. Photomed Laser Surg 24:588-594

11. Ozcelik O, Cenk Haytac M, Kunin A, Seydaoglu G (2008) Improved wound healing by low-level laser irradiation after gingivectomy operations: a controlled clinical pilot study. J Clin Periodontol 35:250-254

12. Skopin MD, Molitor SC (2009) Effects of near-infrared laser exposure in a cellular model of wound healing. Photodermatol Photoimmunol Photomed 25:75-80

13. CDC (1992) 1993 revised classification system for HIV infection and expanded surveillance case definition for AIDS among adolescents and adults. MMWR Recomm Rep 41:1-19

14. Harrison WJ, Lewis CP, Lavy CB (2002) Wound healing after implant surgery in HIV-positive patients. J Bone Joint Surg Br 84:802-806

15. Aird J, Noor S, Lavy C, Rollinson P (2011) The effect of HIV on early wound healing in open fractures treated with internal and external fixation. J Bone Joint Surg Br 93:678-683

16. Davis PA, Corless DJ, Gazzard BG, Wastell C (1999) Increased risk of wound complications and poor healing following laparotomy in HIV-seropositive and AIDS patients. Dig Surg 16:60-67

17. Morandi E, Merlini D, Salvaggio A, Foschi D, Trabucchi E (1999) Prospective study of healing time after hemorrhoidectomy: influence of HIV infection, acquired immunodeficiency syndrome, and anal wound infection. Dis Colon Rectum 42:1140-1144

18. Chen AY, Ohims LA, Stewart MG (1996) Otolaryngologic disease progression in children with HIV. Arch Otolaryngol Head Neck Surg 122:1360-1403

19. Dodson TB (1997) HIV-status and the risk of post-extraction complications. J Dent Res 76:1644-1652

20. Caccuri F, Giagulli C, Bugatti A, Benetti A, Alessandri G, Ribatti D et al (2012) HIV-1 matrix protein p17 promotes angiogenesis via chemokine receptors CXCR1 and CXCR2. Proc Natl Acad Sci U S A 109:14580-145805

21. Albini A, Barillari G, Benelli R, Gallo RC, Ensoli B (1995) Angiogenic properties of human immunodeficiency virus type 1 Tat protein. Proc Natl Acad Sci U S A 92:4838-4842

22. Patsouris E, Katsarou O, Korokolopoulou P (2004) Increased microvascular network in bone marrow of HIV-positive haemophilic patients. HIV Med 5:18-25 